\title{
The use of deep hypothermia and cardiac arrest in the surgical treatment of large and complex intracranial circulation aneurysms
}

\author{
Manuel Cunha e Sá
}

Received: 17 February 2010 / Accepted: 18 February 2010/Published online: 8 April 2010

(C) Springer-Verlag 2010

In this current issue of Acta, Schebesch et al., present their experience with the use of deep hypothermic cardiac arrest (DHCA) in the treatment of 'large and complex' aneurysms of the cerebral circulation over a period of 16 years. The technique was applied in a little less than $4 \%$ of the overall number of aneurysms surgically treated.

DHCA is both an extra-ordinary measure of obtaining circulatory control for longer than usual periods of time whilst securing an effective measure of protecting the brain from an ischemic insult.

This technique has been in the armamentarium of neurosurgeons for decades now and has been the subject of continuous improvements mostly resulting from the increased sophistication of surgical and anesthesiological techniques in use in heart and circulatory bypass peripheral surgery.

Morbidity and mortality associated with DHCA has been reduced significantly making it an invaluable tool to be used with very precise indications. In Neurosurgery it found its application in the treatment of the odd case of giant or more complex intracranial aneurysms for which the estimated time of local circulatory shutdown would surpass the range estimated safe when using conventional brain protection measures.

Large and giant aneurysms can be formidable lesions to tackle with from the surgical standpoint. Given the evidence regarding the dismal natural history of these aneurysms when left untreated, a therapeutic attitude is well grounded. Because most of these more complex lesions may require a long period of temporary local circulatory

\footnotetext{
M. Cunha e Sá $(\bowtie)$

Neurosurgery, Hospital Garcia de Orta,

Almada 2800, Portugal

e-mail: mcunhaesa@gmail.com
}

shutdown, extraordinary measures of cerebral protection may have to be called into action. DHCA is at the extreme end of these protective measures. It entails not only the mobilization of a complex technology and expert team of different surgical specialties not available in all centers but mainly and foremost because it exposes the patient to a non-negligible morbidity and mortality risk.

A balance has to be found between the use of regular protective measures and these extreme solutions. Evolving surgical and neuroradiological technique has significantly changed the process of decision-making in a reasonable number of cases. The use of bypass (prophylactic or therapeutic, low or high flow, conventional or laseroperated), arterial deconstruction or techniques of aneurysmectomy and local re-implantation of arteries has in some cases avoided the need for alternative DHCA. Endovascular treatment with stenting alone or stenting and coiling (although coiling is certainly not the procedure of choice for larger volume aneurysms) has also become an option.

As stated by the authors the use of DHCA is less indicated for anterior circulation aneurysms where exposure is not so much of a problem or to aneurysms for which more elaborate skull base techniques may be of use in securing effective proximal control. Therefore the trend is to restrict the use of DHCA to larger and more complexlooking mostly posterior circulation aneurysms.

What is not accountable in any published series and therefore cannot be taken as a general recommendation for anyone individually tackling with these ferocious lesions and envisaging the use of DHCA, is the personal view one has of each particular case which is going to mold one's own attitude in each situation. This is based on the singular experience of the surgeon in the use of some or all of the above-mentioned techniques and the results achieved with 
each and every one of them. Once the use of a technique is implemented and the confidence grows along with number of cases done it is hard to shy away from using the same strategy repeatedly.

Contemplating different and alternative treatment strategies is essential and so is the acquisition of the (surgical) expertise required in technically implementing them

For this reason the number of aneurysms candidate for a DHCA procedure as compared to the overall number of aneurysms treated with any of the aforementioned techniques can vary significantly from center to center or between surgeons. The mere attribute of complexity to an aneurysm encloses a non-negligible degree of subjectivity.

DHCA remains therefore an important though exceptional way of surgically treating giant and complex intracranial aneurysms. It should only be used in tertiary centers offering a comprehensive approach (both surgical and endovascular) to aneurysm treatment. From the surgical standpoint its use and efficacy should be matched up against deconstructive options, bypass and arterial reimplantation technique.

Despite the fact that endovascular treatment of intracranial aneurysms has become an alternative and rather effective way of treatment the existing evidence still favors surgery as the most effective and long standing way of treatment of giant aneurysms. Statements as to what the future holds in store are no more than promise and manifestations of wishful thinking. Any technique, which undoubtedly demonstrates its superiority over other, should become the gold standard for treatment.

Regardless of the fact that there is no novelty in the material and the conclusions presented I still think it is important that the results of series such as this one be published. They represent a useful piece of information reflecting single center/surgeon experience or in my understanding, 'local or individual evidence based medicine'. 